\title{
Non-invasive Assessment of Vaccine-Induced HPV Antibodies via First-Void Urine
}

\author{
Jade Pattyn*, Severien Van Keer, Laura Téblick, Pierre Van Damme and Alex Vorsters \\ Faculty of Medicine and Health Sciences, Centre for the Evaluation of Vaccination, Vaccine and Infectious Disease Institute \\ (VAXINFECTIO), University of Antwerp, Antwerp, Belgium
}

OPEN ACCESS

Edited by:

lan Frazer,

The University of

Queensland, Australia

Reviewed by:

Rupsa Basu,

TechnoVax Inc, United States

Susana Magadan

University of Vigo, Spain

Julia Brotherton,

VCS Foundation, Australia

*Correspondence:

Jade Pattyn

jade.pattyn@uantwerpen.be

Specialty section:

This article was submitted to Vaccines and Molecular Therapeutics,

a section of the journal

Frontiers in Immunology

Received: 27 March 2020

Accepted: 22 June 2020

Published: 05 August 2020

Citation:

Pattyn J, Van Keer S, Téblick L, Van

Damme $P$ and Vorsters A (2020)

Non-invasive Assessment of Vaccine-Induced HPV Antibodies via

First-Void Urine.

Front. Immunol. 11:1657.

doi: 10.3389/fimmu.2020.01657
The potential of first-void (FV) urine as a non-invasive method to monitor human papillomavirus (HPV) vaccination has been reported, mainly focusing on urine as a sample to assess HPV DNA. Besides HPV DNA, vaccine-induced HPV antibodies originating from cervicovaginal secretions were recently shown to be detectable in FV urine as well. This presents a novel opportunity for non-invasive sampling to monitor HPV antibody status in women participating in large epidemiological studies and HPV vaccine trials. The simultaneous assessment of both HPV infection and immunogenicity on a non-invasive, readily obtained sample is particularly attractive.

Keywords: humaan papillomavirus, HPV vaccination, HPV antibodies, urine, HPV serology

\section{INTRODUCTION}

The evaluation of immunogenicity in HPV vaccine trials relies largely on serology. In the absence of a correlate of protection, it is generally accepted that the presence of high concentrations of vaccine-induced antibodies in serum-greater than those elicited by natural infection-are the best indicators of long-term protection against HPV infection (1). Nevertheless, as cervical cancers typically occur at the cervical transformation zone, it is believed that the presence of mucosal HPV antibodies at the cervix, the site of infection, is critical for vaccine-induced protective immunity. Unlike other mucosal secretions where immunoglobulin A (IgA) predominates, cervicovaginal secretions (CVS) mainly contain IgG transudate from serum and fewer locally produced IgG and secretory IgA (sIgA) (2). Hence, vaccine-induced circulating antibodies are thought to reach the site of infection by transudation at the female genital tract, and by passive exudation at sites of trauma (3). The presence of HPV antibodies at the cervix, using cervicovaginal secretions (CVS) as a proxy, has been reported in a number of studies (4-9) [reviewed in (10)], and just recently in FV urine as well $(11,12)$. Comparable with CVS results $(9)$, there was an approximate 2-log difference in HPV antibody levels between first-void urine and serum (4) and moderate to good correlations between HPV antibody levels in serum and first-void urine were observed $(11,12)$. 


\section{RATIONALE TO DETECT VACCINE-INDUCED HPV ANTIBODIES IN FIRST-VOID URINE}

The presence of HPV-related biomarkers (e.g., HPV DNA) in the urine of women is based on the fact that discharged mucus and debris from exfoliated cells from the female genital organs (including the cervix) accumulate around the urethra opening, between the small labia, and are washed away with the urine flow. Consequently, the initial flow of urine-defined as firstvoid urine-contains significantly more human and HPV DNA than random or mid-stream urine (13-16). Currently, there remains some confusion regarding the definition of first-void urine, which should refer to the initial stream of urine but is sometimes defined as the first urine of the day. Next to the use of first-void urine, other keynotes for improved HPV DNA detection in FV urine were recently summarized (17). Rwanda and Bhutan were the first countries to show the impact of HPV vaccination using optimized urinary HPV DNA testing, confirming the relevance of this sample as representative of the genital tract. These studies also confirmed that FV urine sampling can be successfully implemented in a large cohort study of young adolescent girls $(18,19)$.

Standardized and optimized protocols (including collection, storage, and processing of urine samples) have significantly enhanced the sensitivity of urinary HPV detection and demonstrated good concordance with cervical samples $(16,20$, 21). Furthermore, recent studies indicated that CIN2+ detection using HPV testing of urine shows a sensitivity similar to that of clinician-taken smears or brush-based self-samples $(16,20)$. Because of these promising results, our hypothesis was that CVS-containing HPV-specific antibodies transudated from the circular system-flushed away by the initial urine flow would also harbor HPV-specific antibodies. Recently, a proof of concept study confirmed this hypothesis, and hence the presence of measurable $\mathrm{HPV}$-specific antibodies originating from CVS in FV urine $(11,12)$. In addition, correlations with HPV vaccination status and paired serum samples were found using two different HPV immunoassays not yet specifically designed for urine samples $(11,12)$, making further improvements on the accuracy level possible.

\section{ANALYZING HPV ANTIBODIES IN FIRST-VOID URINE: BENEFITS, DRAWBACKS AND CHALLENGES}

Using urine has several advantages over other more invasive sampling methods. If successful, non-invasive urine sampling could partly replace serum for follow-up of HPV-vaccination and could potentially enhance participation in vaccine trials. Moreover, as urine sampling is non-invasive and does not require trained medical personnel for collection, urine could be of great value for multiple collections at different time points (at home). From the logistical point of view, the possibility to measure both virologic (HPV DNA) and immunological (HPV antibody) end-points in FV urine could ease future surveys and provide major logistical and financial benefits. For example, expansion of HPV vaccination to lower-income countries, particularly with one-or two-dose schedules, may be facilitated by the use of such a tool to assess (early) impact (22). It is clear that, if successful, urinary HPV antibody detection could provide new possibilities for research in settings where serum samples cannot be taken and/or (self-collected) vaginal swabs are less preferred due to for example cultural reasons or a fear of discomfort.

Besides clear benefits, urine also has drawbacks. Because of inter-individual and intra-individual variability, standardization and normalization is essential. The major variations in urinary antibody concentration are determined by the amount of genital secretions washed away by the initial stream of urine, the volume of urine collected (dilution), and the amount of mucus. We are currently investigating the potential of total IgG concentration, total protein concentration, and other potential normalization markers to develop a normalization algorithm. Besides, more research is necessary to investigate the most appropriate buffer to preserve antibodies in first-void and to determine the maximum time between sample collection and detection.

Another challenge using urine consists of generating quantifiable levels of antibodies. Both the amount of antibodies at these mucosal surfaces as well as their presence in urine might be a variable. At the moment we are improving IgG concentration and isolation by pre-treating our urine samples. HPV antibody levels in FV urine were indeed around the detection limit for both the multiplex L1/L2 virus-like particles (VLP)-based ELISA (M4ELISA) and glutathione S-transferase (GST)-L1-based immunoassay (GST-L1-MIA) immunoassay, which complicates the distinction between uronegative and low positive results $(11,12)$. As formal non-inferiority compared to serum is the goal, there is a need for a robust HPV immunoassay that would reach reasonable high sensitivity for urinary HPV antibodies testing. Besides, research to determine the cut-off necessary to make useful conclusions from urine testing has to be established.

Our current research is focused on HPV, however, the idea and technology could be applied to any infection or disease for which monitoring (vaccine-induced) antibody responses is imperative for disease control. Hence, the use of HPV vaccineinduced antibodies could be a model for wider use of FV urine in immunoassays. It is notable, however, that this simplified sample collection is only applicable in females. Although females are a large part of the population, for most of the vaccination trials, both males and females would need to be surveyed. Hence, further research is necessary to develop accurate and feasible non-invasive sampling that can be performed by men. Oral fluid may be such possible non-invasive alternative to serum for HPV antibody surveillance in men and women (23-26).

\section{CONCLUDING REMARKS}

It is clear that the successful development of a technology that measures antibodies in female urine as a way to determine HPV vaccination status, besides HPV DNA status, is very 
promising. Since FV urine collection is less invasive than serum collection, and does not require trained personnel, the validation of such procedure would be of great value for the international HPV research community. In different settings, FV urine could allow vaccine effectiveness and coverage data to be reliably generated post-implementation.

\section{DATA AVAILABILITY STATEMENT}

The original contributions presented in the study are included in the article, further inquiries can be directed to the corresponding author/s.

\section{REFERENCES}

1. Turner TB, Huh WK. HPV vaccines: translating immunogenicity into efficacy. Hum Vaccines Immunother. (2016) 12:14035. doi: $10.1080 / 21645515.2015 .1103936$

2. Mestecky J, Moldoveanu Z, Russell MW. Immunologic uniqueness of the genital tract: challenge for vaccine development. Am J Reprod Immunol. (2005) 53:208-14. doi: 10.1111/j.1600-0897.2005.00267.x

3. Stanley M, Lowy DR, Frazer I. Chapter 12: prophylactic HPV vaccines: underlying mechanisms. Vaccine. (2006) 24(Suppl. 3):S3/106-13. doi: 10.1016/j.vaccine.2006.05.110

4. Draper E, Bissett SL, Howell-Jones R, Waight P, Soldan K, Jit M, et al. A randomized, observer-blinded immunogenicity trial of Cervarix $(R)$ and Gardasil(R) Human Papillomavirus vaccines in 12-15 year old girls. PLoS ONE. (2013) 8:e61825. doi: 10.1371/journal.pone.0061825

5. Schwarz TF, Kocken M, Petaja T, Einstein MH, Spaczynski M, Louwers JA, et al. Correlation between levels of human papillomavirus (HPV)-16 and 18 antibodies in serum and cervicovaginal secretions in girls and women vaccinated with the HPV-16/18 AS04-adjuvanted vaccine. Hum Vaccines. (2010) 6:1054-61. doi: 10.4161/hv.6.12.13399

6. Einstein MH, Levin MJ, Chatterjee A, Chakhtoura N, Takacs P, Catteau G, et al. Comparative humoral and cellular immunogenicity and safety of human papillomavirus (HPV)-16/18 AS04-adjuvanted vaccine and HPV-6/11/16/18 vaccine in healthy women aged 18-45 years: follow-up through Month 48 in a Phase III randomized study. Hum Vaccines Immunother. (2014) 10:345565. doi: 10.4161/hv.36117

7. Nardelli-Haefliger D, Wirthner D, Schiller JT, Lowy DR, Hildesheim A, Ponci F, et al. Specific antibody levels at the cervix during the menstrual cycle of women vaccinated with human papillomavirus 16 virus-like particles. J Natl Cancer Inst. (2003) 95:1128-37. doi: 10.1093/jnci/djg018

8. Scherpenisse M, Mollers M, Schepp RM, Meijer CJ, de Melker HE, Berbers GA, et al. Detection of systemic and mucosal HPV-specific IgG and IgA antibodies in adolescent girls one and two years after HPV vaccination. Hum Vaccines Immunother. (2013) 9:314-21. doi: 10.4161/hv. 22693

9. Kemp TJ, Garcia-Pineres A, Falk RT, Poncelet S, Dessy F, Giannini SL, et al. Evaluation of systemic and mucosal anti-HPV16 and anti-HPV18 antibody responses from vaccinated women. Vaccine. (2008) 26:360816. doi: 10.1016/j.vaccine.2008.04.074

10. Pattyn J, Van Keer S, Tjalma W, Matheeussen V, Van Damme P, Vorsters A. Infection and vaccine-induced HPV-specific antibodies in cervicovaginal secretions. A review of the literature. Papillomavirus Res. (2019) 8:100185. doi: 10.1016/j.pvr.2019.100185

11. Pattyn J, Panicker G, Willhauck-Fleckenstein M, Van Keer S, Téblick L, Pieters Z, et al. Comparison of a VLP-based and GST-L1-based multiplex immunoassay to detect vaccine-induced HPV-specific antibodies in first-void urine. J Med Virol. (2020). doi: 10.1002/jmv.25841. [Epub ahead of print].

12. Van Keer S, Willhauck-Fleckenstein M, Pattyn J, Butt J, Tjalma WAA, Van Ostade X, et al. First-void urine as a non-invasive liquid biopsy source to detect vaccine-induced human papillomavirus antibodies

\section{AUTHOR CONTRIBUTIONS}

All authors listed have made a substantial, direct and intellectual contribution to the work, and approved it for publication.

\section{FUNDING}

JP is supported by a Ph.D. fellowship of the Royal Belgian Academy of Medicine (GSK grant). SV is supported by a junior postdoctoral fellowship of the Research Foundation Flanders (1240220N). originating from cervicovaginal secretions. J Clin Virol. (2019) 117:11-18. doi: 10.1016/j.jcv.2019.05.004

13. Vorsters A, Van Damme P, Clifford G. Urine testing for HPV: rationale for using first void. BMJ. (2014) 349:g6252. doi: 10.1136/bmj.g6252

14. Senkomago V, Des Marais AC, Rahangdale L, Vibat CR, Erlander MG, Smith JS. Comparison of urine specimen collection times and testing fractions for the detection of high-risk human papillomavirus and highgrade cervical precancer. J Clin Virol. (2016) 74:26-31. doi: 10.1016/j.jcv.2015. 11.005

15. Pathak N, Dodds J, Zamora J, Khan K. Accuracy of urinary human papillomavirus testing for presence of cervical HPV: systematic review and meta-analysis. BMJ. (2014) 349:g5264. doi: 10.1136/bmj.g5264

16. Leeman A, Del Pino M, Molijn A, Rodriguez A, Torne A, de Koning $\mathrm{M}$, et al. HPV testing in first-void urine provides sensitivity for CIN2+ detection comparable with a smear taken by a clinician or a brush-based self-sample: cross-sectional data from a triage population. BJOG. (2017) 124:1356-63. doi: 10.1111/1471-0528.14682

17. Pattyn J, Van Keer S, Téblick L, Van Damme P, Vorsters A. HPV DNA detection in urine samples of women: "an efficacious and accurate alternative to cervical samples?". Expert Rev Anti Infect Ther. (2019) 17:7557. doi: $10.1080 / 14787210.2019 .1668776$

18. Franceschi S, Chantal Umulisa M, Tshomo U, Gheit T, Baussano I, Tenet V, et al. Urine testing to monitor the impact of HPV vaccination in Bhutan and Rwanda. Int J Cancer. (2016) 139:518-26. doi: 10.1002/ijc.30092

19. Tshomo U, Franceschi S, Tshokey T, Tobgay T, Baussano I, Tenet V, et al. Evaluation of the performance of Human Papillomavirus testing in paired urine and clinician-collected cervical samples among women aged over 30 years in Bhutan. Virol J. (2017) 14:74. doi: 10.1186/s12985-017-0744-2

20. Arbyn M, Peeters E, Benoy I, Vanden Broeck D, Bogers J, De Sutter P, et al. VALHUDES: a protocol for validation of human papillomavirus assays and collection devices for HPV testing on self-samples and urine samples. J Clin Virol. (2018) 107:52-6. doi: 10.1016/j.jcv.2018. 08.006

21. Cuzick J, Cadman L, Ahmad AS, Ho L, Terry G, Kleeman M, et al. Performance and diagnostic accuracy of a urine-based human papillomavirus assay in a referral population. Cancer Epidemiol Biomark Prev. (2017) 26:1053-9. doi: 10.1158/1055-9965.EPI-16-0960

22. Franceschi S, Clifford GM, Baussano I. Options for design of real-world impact studies of single-dose vaccine schedules. Vaccine. (2018) $36(32 \mathrm{Pt}$ A):4816-22. doi: 10.1016/j.vaccine.2018.02.002

23. Parker KH, Kemp TJ, Pan Y, Yang Z, Giuliano AR, Pinto LA. Evaluation of HPV-16 and HPV-18 specific antibody measurements in saliva collected in oral rinses and merocel $\mathbb{R}$ sponges. Vaccine. (2018) 36:2705-11. doi: 10.1016/j.vaccine.2018.03.034

24. Handisurya A, Schellenbacher C, Haitel A, Senger T, Kirnbauer R. Human papillomavirus vaccination induces neutralising antibodies in oral mucosal fluids. Br J Cancer. (2016) 114:409-16. doi: 10.1038/bjc.20 15.462

25. Rowhani-Rahbar A, Carter JJ, Hawes SE, Hughes JP, Weiss NS, Galloway $\mathrm{DA}$, et al. Antibody responses in oral fluid after administration of 
prophylactic human papillomavirus vaccines. J Infect Dis. (2009) 200:14525. doi: $10.1086 / 606026$

26. Louie KS, Dalel J, Reuter C, Bissett SL, Kleeman M, AshdownBarr L, et al. Evaluation of dried blood spots and oral fluids as alternatives to serum for human papillomavirus antibody surveillance. mSphere. (2018) 3: e00043-18. doi: 10.1128/mSphere.00 043-18

Conflict of Interest: PV and AV are co-founders and former board members of Novosanis (Belgium), a spin-off company of the University of Antwerp that produces a device for collection and preservation of first void urine.
The remaining authors declare that the research was conducted in the absence of any commercial or financial relationships that could be construed as a potential conflict of interest.

Copyright (๑ 2020 Pattyn, Van Keer, Téblick, Van Damme and Vorsters. This is an open-access article distributed under the terms of the Creative Commons Attribution License (CC BY). The use, distribution or reproduction in other forums is permitted, provided the original author(s) and the copyright owner(s) are credited and that the original publication in this journal is cited, in accordance with accepted academic practice. No use, distribution or reproduction is permitted which does not comply with these terms. 